\title{
Effect of Conditions for Gas Recirculation on Divertor Operation in ITER
}

\author{
A.S. Kukushkin ${ }^{1, *}$, H.D. Pacher ${ }^{2}$, V. Kotov ${ }^{3}$, D. Reiter ${ }^{3}$, D. Coster ${ }^{4}$, G.W. Pacher $^{5}$ \\ ${ }^{1}$ ITER IT, Garching, Germany \\ ${ }^{2}$ INRS-EMT, Varennes, Québec, Canada \\ ${ }^{3}$ FZ Jülich, Jülich, Germany \\ ${ }^{4}$ Max-Planck Institut für Plasmaphysik, Garching, Germany \\ ${ }^{5}$ Hydro-Québec, Varennes, Québec, Canada \\ E-mail contact of the main author: Andrei.Kukushkin@iter.org
}

\begin{abstract}
The latest results of B2-Eirene modelling of ITER divertor operation are presented. The operational window is further explored with an improved model of the neutral transport, the effect of gas leaks between the divertor cassettes is assessed, and the sensitivity of the results to features of the divertor geometry and to the gas puffing arrangement is analysed. The presence of neutral-neutral collisions in the model makes the results rather insensitive to the detail of the divertor shape. The analysis shows that the effect of the gas leak on the divertor performance in ITER is weak and therefore inter-cassette sealing may be not critical.
\end{abstract}

\section{Introduction}

One of the important aspects of ITER divertor design, not addressed in previous modelling studies, is the presence of gas leaks through the divertor structures. The ITER divertor consists of 54 modules (cassettes) [1] that are to be assembled remotely inside the vacuum vessel. For technical reasons the poloidal gaps between the cassettes cannot be sealed perfectly, and therefore unintended particle fluxes will occur between the divertor and the gas volume inside the cassette. The primary aim of this paper is to explore the effect of these fluxes on the performance of the ITER divertor. Furthermore, the sensitivity of the divertor operation to the position of the separatrix strike points on the targets and to particular features

\footnotetext{
* Present affiliation: ITER Organisation, Cadarache, France
} 
of the target shape and arrangement of gas puff is re-considered by means of the recently improved model [2] now routinely used for ITER analysis.

In the present study, we use the B2-Eirene code package version solps4.2 [2] which, in particular, relies on advanced features of Eirene $^{1}[3]$, such as a non-linear model of neutral particle transport, including neutral-neutral and molecule-ion collisions. For the present study, we take the whole inner surface of ITER to be covered by carbon. The other model assumptions are those of [2]. In particular, we fix the plasma power entering the scrape-off layer (SOL) at $100 \mathrm{MW}$, fix the D ion flow from the core across the core-edge interface (CEI) at $17 \mathrm{~Pa} \cdot \mathrm{m}^{3} \mathrm{~s}^{-1}$, and vary the density by varying the flux injected by gas puffing. The $\mathrm{D}_{2}$ gas is puffed from the top (except for one variant), and pumped via the duct in the private-flux region (PFR) at the bottom of the chamber and via the cassette leaks. The plasma consists of $\mathrm{D}$ (representing both $\mathrm{D}$ and $\mathrm{T}), \mathrm{He}$, and $\mathrm{C}$ ions and atoms, and $\mathrm{D}_{2}$ molecules.

The model taking into account the gas leaks between the divertor components is described in section 2, and the results upon application to ITER are shown in section 3. The sensitivity of the modelling results to the shape of the bottom of the divertor and to the shift of the separatrix strike points on the targets are analysed in sections 4 and 5, the effect of a midplane gas puff is described in section 6 , and the conclusions are given in section 7.

\section{Model of Gas Leaks in the Divertor}

A cartoon of the particle flows (He or D - they are treated in similar fashion in the model) in the divertor region is shown in Fig. 1 . Here $\Gamma_{p}$ is the flux to the pump, $\Gamma_{d}$ the flux from the PFR into the duct, $\Gamma_{\mathrm{i}}$ the ion flux from the core to the SOL, $\Gamma_{\mathrm{n}}$ the neutral flux from the SOL to the core, $\Gamma_{\mathrm{c}}$ the core fuelling (for D) or production (for He). We model two kinds of leaks: from the cassette body to the SOL at the baffle, $\Gamma_{\mathrm{b}}$, and from the PFR to the cassette body at

\footnotetext{
${ }^{1}$ see also: http://www.eirene.de
} 
the "V"s in the divertor corners, $\Gamma_{\mathrm{v}}$. The leaks are characterized by the gas conductance of the corresponding gaps and the pressure difference. In our calculations, we do not model the neutral transport within the cassette body - modelling of this essentially three-dimensional volume would be computationally too expensive. Instead we specify the reflux at the baffle due to the gaps as an additional gas puff, $\Gamma_{\mathrm{b}}$, and the pumping due to the gaps as extra particle absorption at the "V", $\Gamma_{\mathrm{v}}$. This scheme is justified by the observation that, in our modelling the gas pressure at the "V"s, $\mathrm{p}_{\mathrm{v}}$, is always considerably (a factor 3 or more) higher than the pressure at the duct entrance, $p_{d}$, which is in turn higher than the pressure inside the cassette body, $\mathrm{p}_{\mathrm{c}}$ (which is not calculated in our model). This will always occur because the pumping from PFR under the dome is the major contribution to the pumping. The pressure at the baffle $\mathrm{p}_{\mathrm{b}}$ is much lower than $\mathrm{p}_{\mathrm{d}}$, so that for any reasonable gas conductance of the duct, simple estimates give $p_{c}>p_{b}$. These relations of the gas pressure inside the cassette to that at different locations in the divertor determine the direction of particle flows through the gaps, and we use them to simplify the model.

In these conditions, a natural measure for the leaks through the "V"s is the ratio of the "reduced" pumping speed through the "V" surface to the sum of this pumping speed and that through the pumping duct. Here, "reduced" means the pumping speed estimated for the same gas temperature, so that the leakage factor $\alpha_{v}=\left(\xi_{v} \cdot A_{v}\right) /\left(\xi_{d} \cdot A_{d}+\xi_{v} \cdot A_{v}\right)[4]$ where $\xi$ stands for the particle absorption at, and A for the area of, the corresponding surface. For most of the runs with the V-leak, $\alpha_{\mathrm{v}}=0.2$ was selected, which results in $\Gamma_{\mathrm{v}} \sim \Gamma_{\mathrm{d}}$ because $\mathrm{p}_{\mathrm{v}}$ is several times $\mathrm{p}_{\mathrm{d}}$. Typical gap sizes for the ITER design without specific sealing measures are 54 toroidal gaps, $2 \mathrm{~m}$ long, $5 \mathrm{~mm}$ wide and $5 \mathrm{~cm}$ deep [5]. Accurate evaluation of $\alpha_{\mathrm{v}}$ would require Monte-Carlo neutral calculations, but, taking $\mathrm{p}_{\mathrm{c}}<<\mathrm{p}_{\mathrm{v}}$, one can estimate a value $\alpha_{\mathrm{v}}<$ $0.01[5,6]$. An accurate evaluation of such a tiny leak with a Monte-Carlo model would be challenging, and the results would probably not justify the time invested in such an effort. 
Instead, we assume a monotonic behaviour of the effect of the leaks with increasing gap width. Comparing the results with $\alpha_{\mathrm{v}}=0$ (no leaks) and 0.2 , we are justified in asserting that the effect of these leaks is bracketed by the calculations. If sealing measures reduced the gap size to $1 \mathrm{~mm}$, use of expressions such as those of [6] would indicate that the gaps would then be entirely negligible.

A natural measure of the leak from the cassette to the SOL through the baffle is the ratio $\alpha_{b}=$ $\Gamma_{\mathrm{b}} /\left(\Gamma_{\mathrm{b}}+\Gamma_{\mathrm{p}}\right)$ (note that there is no singularity here when $\left.\Gamma_{\mathrm{p}}=0\right)$. If the gas pressure inside the cassette body $\mathrm{p}_{\mathrm{c}}$ were uniform, this would be the ratio of the gas conductance of the leaks in the baffle to the sum of this conductance with that of the downpipe to the pump. The desired value of $\alpha_{b}$ is achieved in the course of a calculation via adjustment of the additional puffed flux $\Gamma_{\mathrm{b}}$. The value $\alpha_{\mathrm{b}}=0.3$, that is, $\Gamma_{\mathrm{b}} \cong 0.5 \Gamma_{\mathrm{p}}$, corresponds to the cassette design without sealing [5]. In the model, the reflux is usually distributed uniformly over the baffle (inner and outer) region, and calculations show a weak sensitivity of the results to this distribution. Note that more than an order of magnitude difference between the values of $\alpha_{b}$ and $\alpha_{v}$ for the same width of the gaps in the baffle and "V" areas is quite natural: $\alpha_{b}$ reflects the competition between the gas conductance of the gaps and that of the downpipes, whereas $\alpha_{\mathrm{v}}$ compares the conductance of the gaps with that of the grill-like structure at the duct inlet, which is much higher.

Since the atomic masses of $\mathrm{He}$ and $\mathrm{D}_{2}$ are the same, and they are in equilibrium with the walls within the cassette body, it is reasonable to assume that the pressure distribution there is similar for He and $\mathrm{D}_{2}$. Then for each plasma component

$$
\Gamma_{p}=\Gamma_{d}+\Gamma_{v}-\Gamma_{b}, \quad \Gamma_{b}=\alpha_{b}\left(\Gamma_{p}+\Gamma_{b}\right)=\alpha_{b}\left(\Gamma_{d}+\Gamma_{v}\right), \quad \Gamma_{p}=\left(1-\alpha_{b}\right)\left(\Gamma_{d}+\Gamma_{v}\right)
$$


The helium ion flux $\Gamma_{\mathrm{i}}$ from the core to the SOL, equal to the sum of the fusion $\mathrm{He}$ production rate in the core and the neutral flux from the SOL to the core, $\Gamma_{\mathrm{i}}=\Gamma_{\mathrm{c}}+\Gamma_{\mathrm{n}}$, is specified at the CEI, and this value is adjusted in the course of the calculation.

\section{Effect of the Leaks on Divertor Performance}

The operational space of the ITER divertor is multi-dimensional [7], where some dimensions represent the engineering parameters ( $\mathrm{q}_{\mathrm{pk}}$, particle throughput to the pump), some the control parameters (gas puffing rate and pumping speed), and some characterize the interface with the core plasma (power input to the SOL, separatrix density, etc.). Constraints stemming from design limitations and physical considerations delineate an operational window in this operational space. A detailed study of the operational space of the ITER divertor was presented in [7], although with a simplified, linear model of the neutral particle transport. From that study we know that the most important parameters determining the divertor performance are the power input to the SOL and the neutral gas pressure in the PFR, $\mathrm{p}_{\mathrm{DT}}$. In the present paper we vary $\mathrm{p}_{\mathrm{DT}}$ by varying the gas puffing rate and keeping the pumping speed and the input power constant, thus obtaining density scans. In particular, we consider the upper limit of the operational window at the high $\mathrm{p}_{\mathrm{DT}}$ side to be the onset of full plasma detachment in the inner divertor leg, since beyond this point the neutral reflux to the core increases more rapidly.

In order to determine the effect of particle reflux, we performed three density scans for the values of $\alpha_{b}=0,0.15$, and 0.5 , keeping $\alpha_{v}=0.2$. In some of the figures, the position of the transition to detached operation of the inner divertor is indicated (see [8]). Fig. 2 shows the variation of peak power load of the target $\mathrm{q}_{\mathrm{pk}}$ with the neutral pressure in the PFR. No significant change occurs when $\alpha_{b}$ or $\alpha_{v}$ is varied - that is, the power load remains strongly correlated with the neutral pressure in the PFR, as in $[2,7,8]$. The maximum of the plasma temperature along the targets decreases (Fig. $3 a$ and $3 b$ ) when the V-leak is 
introduced (compare the curve for $\alpha_{v}=0.2, \alpha_{b}=0$ with the standard configuration), and this reduction progresses along with the increase of $\alpha_{b}$. This occurs because, to maintain the same average pressure at the interface to the PFR, the throughput must be increased when the additional pumping through the "V"s is introduced, leading to additional recycling in the main chamber. The variation of the neutral density at the top part of the divertor plate is determined by the trade-off between the additional pumping due to the "V"s and this additional recycling, and the additional recycling dominates. The increased neutral pressure there leads to a lower plasma temperature at the target. When the reflux neutrals are introduced (variation of $\alpha_{b}$ from 0 to $75 \%$ ) in addition at the baffles, the temperature at the top of the targets, where $T_{\max }$ is located if there is no reflux, decreases even further, Fig. 3c.

The effect of the particle reflux on the core interface parameters is shown in Fig. 4. Here the average ion density at the separatrix and the neutral influx into the core through it are shown for D (fuelling) and He (helium removal). For D, both the ion density at the separatrix and the neutral influx to the core increase slightly in the presence of the leaks (a clear progression from the no leak case to that having the leak at the "V"s and finally to the case at higher reflux), mostly because the throughput (and therefore the fuelling) increases for the same $\mathrm{p}_{\mathrm{DT}}$ and therefore the recycling in the main chamber increases also.

To facilitate the assessment of the influence of the gaps, we introduce an effective engineering pumping speed which is proportional to the particle absorption fraction $\xi$, as specified in the model, times the absorbing area [4], corrected for the particle reflux fraction. This is obtained as follows. Pumping is given by an absorption $\xi_{\mathrm{d}}$ at the pump duct, pumping through the "V"s by an absorption $\xi_{\mathrm{v}}$, and the particle reflux is characterised by $\alpha_{\mathrm{b}}$. Using Eq. (1), one can combine these parameters to get the pumping speed in the presence of the gas leaks in the divertor structures, normalised to the value without these: 


$$
S_{n} \equiv S_{p} / S_{d}=\left(1+\frac{A_{v} \xi_{v}}{A_{d} \xi_{d}}\right)\left(1-\alpha_{b}\right)=\frac{1-\alpha_{b}}{1-\alpha_{v}}
$$

Here $S_{p}$ is the effective pumping speed relating the total throughput to the pump to the neutral pressure in the divertor (if the latter were uniform and the neutral temperature were standard room temperature), $S_{d}$ is the nominal pumping speed at the duct entrance.

The helium-related quantities in Fig. 4 are scaled with this effective engineering pumping speed $S_{n}$ because, in the absence of profile effects, at equal helium production rate and therefore throughput, the helium related quantities should be inversely proportional to the pumping speed, and previous studies [7] have confirmed that this remains the major effect for the complete calculation. Indeed, this continues to hold for the present results: whereas the He neutral flux is quite different for cases with and without leaks at the "V"s and/or reflux, it superposes well when scaled with $S_{n}$ (Fig. 4d). The scaled helium density at the separatrix, $\mathrm{n}_{\text {He_sep }}$, however, decreases with reflux, Fig. $4 \mathrm{c}$, which appears counter-intuitive at first. This becomes understandable if one considers a qualitative picture of helium flows in the SOL and divertor [7,9] as shown in Fig. 5. The thermal force impedes the helium ion flow along the hotter part of the SOL, close to the separatrix, so that most of the helium ions reach the targets further away from the separatrix. Since the helium-helium interactions are insignificant for the transport, the problem becomes linear and the helium density profile can be represented as a sum of two components corresponding to different sources: one from the fusion production in the core, another from the reflux. The reflux component contributes less to the separatrix density since the bulk of the neutral influx is ionized in the outer SOL where the ions can flow back to the targets, and this additional source is compensated in the model by increased pumping in the PFR $\left(\Gamma_{\mathrm{p}}=\Gamma_{\mathrm{c}}\right.$ for He). Therefore re-direction of some part of the helium throughput at the divertor bottom to the reflux channel should cause a reduction of $\mathrm{n}_{\mathrm{He} \_ \text {sep }}$, as we see in our calculations. Fig. 6a shows a comparison of the radial profiles of helium ion density in the SOL taken at the outer mid-plane from two special runs. In the first one, the 
helium reflux was set to zero - that is, all the helium was supplied from the core,- whereas in the second run the core source was switched off and the same amount of helium was puffed from the baffle. As can be seen from Fig. 6a, the second case shows lower helium density at the separatrix, demonstrating the effect.

The helium enrichment at given $\mathrm{p}_{\mathrm{DT}}$, helium production rate, and pumping speed (Fig. 6b) is lower by $\sim 30 \%$ in the presence of the gaps (equivalent to a higher required DT fuelling at constant He production rate by the same amount). Working at the same enrichment would require a $\sim 20 \%$ lower $\mathrm{p}_{\mathrm{DT}}$, undesirable because of the increase of power load and helium density then. However, the effect is not large, even with the strongly oversized leakage $\left(\alpha_{v}=\right.$ 0.2 instead of 0.01$)$.

The effects of the leaks are therefore either relatively small (peak power load, helium flux, DT neutral flux to the core, helium enrichment) or beneficial (helium ion density).

\section{Sensitivity to the Target Shape}

Since no negative effect of the gas leaks around the divertor corners was found (previous section), and these leaks should affect the tightness of the "V"-shaped parts of the divertor plates to neutral transport, it is natural to re-assess the role of the latter in the divertor performance in the framework of the improved model of the neutral particle transport. In order to do this, we consider four different modifications of the divertor shape, as shown in Fig. 7, ranging from very open corners (F31) to a deep "V" (F34).

The result is illustrated in Fig. 8. We see no significant difference in the parameters determining the core fuelling $\left(\Gamma_{\mathrm{DT} \_} \mathrm{n}_{-} \mathrm{sep}, \mathrm{n}_{\mathrm{DT} \_ \text {sep }}\right)$, or the helium removal $\left(\mathrm{n}_{\mathrm{He} \_ \text {sep }}, \Gamma_{\mathrm{He} \_} \mathrm{n}_{-} \mathrm{sep}\right)$. The target power load ( $\left.\mathrm{q}_{\mathrm{pk}}\right)$ can decrease somewhat (F34 vs. F12), but only by less than $40 \%$, and this corresponds to a shift of the operational window by approximately $20 \%$ towards lower values of $\mathrm{p}_{\mathrm{DT}}$. 
This relative insensitivity to geometry differs from previous studies [10] which used the linear neutral transport model that took into account neither neutral-neutral collisions nor collisions of molecules with plasma ions. Therefore the neutral particles leaving the plasma were allowed to travel freely within the PFR, and their trajectories were determined solely by collisions with the solid surfaces. So the shape of these surfaces (not $\mathrm{p}_{\mathrm{DT}}$ !) played an important role in the transport of neutrals. With the present model, the neutrals are scattered during the neutral-neutral collisions, and their mean-free-path is around $1 \mathrm{~cm}$ for typical pressures. As a result, the shape of the divertor structures does not affect their transport provided that $\mathrm{p}_{\mathrm{DT}}$ is the same, and therefore the effect of geometry changes is much weaker with this more complete model.

\section{Sensitivity to the strike-point position}

The positions of the separatrix strike-points on the divertor targets can only be kept with certain accuracy, and higher accuracy imposes more severe demands on the control system. The studies shown in the previous section (progression from F12 to F32) indicate that a shift of the strike-points towards the floor of the PFR would not affect the divertor performance significantly; this shift is limited only by the heat load on the dome. To explore the effect of the upward shift of the strike-points, we selected three configurations including the reference geometry, Fig. 9. The shifted configurations were in fact produced in the simulations by keeping the same plasma equilibrium and shifting the divertor down by 12 and $24 \mathrm{~cm}$ (F21 and F20, respectively). Therefore the geometry of the SOL remains the same, and the effect of the variation of the divertor geometry should become apparent.

The results are shown in Fig. 10. As for the divertor shape variation, no significant difference in the performance parameters is seen, other than a slight $(\sim 20 \%)$ shift of the operational window to lower neutral pressure for the F20 geometry and a 20\% increase in the separatrix DT density. To describe the results at the same operating point relative to divertor 
detachment, we therefore use a normalised value of the neutral pressure, $p_{D T}{ }^{*}=p_{D T} f_{S}^{-1}$, where $f_{s}=0.78$ for F20 and 1 for the other geometries considered. With this normalisation, the operational parameters for all three geometries follow similar curves, Fig. 10, except for a slight increase in $\mathrm{n}_{\text {He_sep }}$ in F20 - the helium neutralisation zone on the targets (see Fig. 5) becomes here well screened from the pump duct and this impedes He pumping.

\section{Mid-plane gas puff}

In another exploration of modification of the gas recirculation pattern in the edge, we study the effect of moving the location of the gas puff from the top of the machine to the outer midplane (OMP). One might naively expect the latter case to have much higher fuelling efficiency for the core plasma since the SOL in the OMP is almost an order of magnitude narrower than at the top (where there is considerable flux expansion because the secondary Xpoint is close to the first wall in ITER). However, our calculations show that this is not the case.

Fig. 11 compares the neutral flux to the core for the two puff locations. One can see a moderate (a factor 2 to 3 ) increase of the fuelling efficiency when the gas is puffed from the OMP, which would still not suffice to fuel the central plasma of ITER by gas puffing alone [11]. The increase remains moderate for the following reason. Since the secondary X-point is close to the first wall, the magnetic pitch angle at the top of the chamber is small, so that for a given poloidal distance the temperature drop due to parallel heat conduction is larger there than elsewhere on the magnetic surface. The parallel heat transport is therefore not sufficient to maintain a nearly constant temperature when the gas is puffed there, and a cool plasma region forms in front of the gas injector as seen in Fig. 12. Consequently, the neutral penetration for top puffing is better than one might have expected. The density profiles in front of the puffing slots are similar. The peak power at the divertor plate $\mathrm{q}_{\mathrm{pk}}$ does not change 
for the same p $_{\mathrm{DT}}$ (Fig. 13a). For the OMP puff, the mid-plane ion temperature decreases by about $10 \%$ at the OMP puff location, and this explains the reduction of the helium ion density at the separatrix, Fig. 13b. Indeed, the thermal force balances the He pressure gradient when $\mathrm{n}_{\mathrm{MP}} / \mathrm{n}_{\text {div }} \sim\left(\mathrm{T}_{\mathrm{MP}} / \mathrm{T}_{\text {div }}\right)^{3.5}$ [9]. Since $\mathrm{T}_{\text {div }}$ and $\mathrm{n}_{\text {div }}$ do not change, a $10 \%$ reduction of $\mathrm{T}_{\mathrm{MP}}$ gives rise to a $50 \%$ reduction in $\mathrm{n}_{\mathrm{MP}}$. Reduction of $\mathrm{He}$ ion density in the SOL leads to a corresponding reduction of the helium atom flux recycling at the first wall and this reduces the helium influx to the core, Fig. 13c. The reduction of $\mathrm{n}_{\mathrm{He}}$ and $\Gamma_{\mathrm{n}_{-} \mathrm{He} \_ \text {sep }}$ by a factor 1.5 to 2 is beneficial, but would be accompanied with an increased bombardment of the gas inlet area by energetic charge exchange neutrals, which may dominate erosion of non-carbon materials (not considered in the present paper).

\section{Conclusions}

We have further developed the computational model of the edge plasma in ITER, taking into account particle leaks through the gaps between the divertor cassettes. First results indicate no major negative effect of the gaps on the divertor performance, although the parasitic flows caused by these gaps can be comparable with the pumping throughput. In the presence of the leaks, the dependence of the peak target power load on the neutral gas pressure in the divertor remains unchanged and the core fuelling efficiency remains the same. The helium density at the separatrix decreases when the gaps are present because, at the same pumping speed at the pump, a fraction of the helium flux from the PFR is redirected to the baffle area via the leaks and contributes less to the helium density build-up at the separatrix. However, the helium enrichment decreases by $30 \%$, i.e. the DT throughput is larger by this amount at the same helium production rate. Since the presently assumed values $\left(\alpha_{v}=0.2, \alpha_{b}=0.5\right)$ correspond to gaps at least 2 times wider than the design value of $5 \mathrm{~mm}$, and the effects are relatively small, sealing the inter-cassette gaps in ITER may not be a high priority issue. 
The calculations reported in this paper confirm our previous observation [4] that the more comprehensive, non-linear model of the transport of the neutral particles [2] leads to a much weaker sensitivity of the results to particular features of the divertor geometry. In particular, it is not as important to maintain tightly sealed, pronounced V-shapes at the corners of the divertor targets close to the separatrix strike points as had been suggested earlier on the basis of the linear neutral model [10] because these geometric effects are attenuated by neutralneutral collisions. This increases the flexibility, facilitating optimisation of both the divertor design and the plasma configuration in ITER. An upward deviation of the separatrix strikepoints on the targets from their nominal positions by $20 \mathrm{~cm}$ is found tolerable, and their downward deviation is determined not by divertor performance but rather by the acceptable heat load on the dome.

The gain in the core fuelling efficiency by changing the location of the main gas puff from the top to the outer mid-plane would be a factor 2 to 3 , still insufficient to provide the required core fuelling by gas puffing alone. The decision is a trade-off between, on the one hand, the improvement of core fuelling efficiency (factor 2-3) and helium removal (factor 2) and on the other, likely increased wall erosion by energetic charge exchange neutrals near the mid-plane puffing location. Further dedicated modelling is needed to quantify these effects.

Further systematic work is needed and will be performed to augment these observations. In particular, the effect of gas leaks through the divertor dome should be considered, together with a possible effect of the leaks on the gas pressure inside the divertor cassettes and so on the pumping efficiency, before a final recommendation on inter-cassette sealing can be given.

\section{Acknowledgement}


This report was prepared as an account of work undertaken within the framework of ITER Transitional Arrangements (ITA). These are conducted by the Participants: China, the European Atomic Energy Community, India, Japan, Korea, the Russian Federation, and the United States of America, under the auspices of the International Atomic Energy Agency. The views and opinions expressed herein do not necessarily reflect those of the Participants to the ITA, the IAEA or any agency thereof. Dissemination of the information in this paper is governed by the applicable terms of the former ITER EDA Agreement. This work was partly supported by the EFDA Technology Workprogram 2005-2006 TW5-TPP-ITERNEUT. 


\section{References}

[1] G. Janeschitz, A. Antipenkov, G. Federici, et al., Nucl. Fusion 42 (2002) 14.

[2] A.S. Kukushkin, H.D. Pacher, V. Kotov, et al., Nucl. Fusion 45 (2005) 608.

[3] D. Reiter, M. Baelmans, P. Börner, Fusion Sc. Tech. 47 (2005) 172.

[4] A.S. Kukushkin, H.D. Pacher, V. Kotov, et al., "Effect of the Dome on Divertor Performance in ITER", $17^{\text {th }}$ PSI Conference, Hefei, 2006 (to appear in J. Nucl. Mater.)

[5] C. Lowry (ITER), private communication, 2006.

[6] W. Steckelmacher, J. Phys. D: Appl. Phys. 11 (1978) 473.

[7] A.S. Kukushkin, H.D. Pacher, G.W. Pacher, et al., Nucl. Fusion 43 (2003) 716.

[8] H.D. Pacher, A.S. Kukushkin, G. W. Pacher, et al., "Effect of the Tokamak Size in Edge Transport Modelling and Implications for DEMO”, 17th PSI Conference, Hefei, 2006 (to appear in J. Nucl. Mater.)

[9] S.I. Krasheninnikov, A.S. Kukushkin, T.K. Soboleva, Nucl. Fusion 31 (1991) 1455.

[10] A. S. Kukushkin, H.D. Pacher, Plasma Phys. Control. Fusion 44 (2002) 931.

[11] G.W. Pacher, H.D. Pacher, G Janeschitz, et al., Plasma Phys. Contr. Fusion 46 (2004) A257. 
Figure captions

Fig. 1. Schematic of the particle flow in the divertor region of ITER

Fig. 2. Peak power load vs. $p_{\mathrm{DT}}$ for different values of the particle reflux fraction $\alpha_{b}$. Two exploratory series of runs where one of $\alpha_{b}, \alpha_{v}$ was varied whereas the other and the total D content in the model were kept constant are also shown.

Fig. 3. Maximum electron temperature in the inner (a) and outer (b) divertor vs. $p_{D T}$ for different values of the particle reflux fraction $\alpha_{b}$. Fig. $3 c$ shows the radial profile of electron temperature at the outer target for $\mathrm{p}_{\mathrm{DT}}=13 \mathrm{~Pa}$.

Fig. 4. Ion density of D (a) and He (c), and neutral flux to the core of D (b) and He (d) vs. $p_{D T}$ for different values of particle reflux fraction $\alpha_{b}$ for the same series as Fig. 2 and 3. The helium-related quantities are scaled appropriately with the normalised effective pumping speed $S_{n}$ (see text and [8], values indicated in the legend).

Fig. 5. Schematic of helium flow in the SOL and divertor region (unfolded on a rectangular area). Left and right edges represent the targets, upper edge the outer wall.

Fig. 6. (a) radial profiles of helium ion density in the SOL (outer mid-plane). (b) helium enrichment vs. $p_{\mathrm{DT}}$ for different values of particle reflux fraction $\alpha_{\mathrm{b}}$. The enrichment is scaled appropriately with the normalised effective pumping speed $S_{n}$ (see text and [8]). Legend as in Fig. 4.

Fig. 7. Variation of divertor geometry used in the re-exploration on the effect of the "Vshapes" near the separatrix strike points. F12 marks our standard modelling configuration, F28, F31, and F32 are more open, and F34 more tight. Dotted lines indicate partially transparent surfaces at the entrance to the PFR.

Fig. 8. Peak power (a), D neutral influx into the core (b) and He ion density at the separatrix (c) vs. $p_{\mathrm{DT}}$ for different shapes of the divertor bottom (see Fig. 7). $\mathrm{S}_{\mathrm{n}}=0.83$ for F32 and 1 for the other configurations.

Fig. 9. Variation of the separatrix strike-point position on the targets used in the exploration. F12 is the reference geometry, F21 corresponds to an upward shift of $12 \mathrm{~cm}$ and F20 of 24 $\mathrm{cm}$.

Fig. 10. Peak power (a), D neutral influx into the core (b) and He ion density at the separatrix (c) vs. normalised pressure in PFR, $\mathrm{p}_{\mathrm{DT}}{ }^{*}$, for different positions of the separatrix strike-points on the targets (see Fig. 9).

Fig. 11. Influx of D neutrals into the core (core fuelling rate) vs. $p_{D T}$ for different locations of the gas puff. "trans" indicates incipient detachment of inner divertor (see text).

Fig. 12 Radial profiles of the electron temperature (a) and electron density (b), mapped along the magnetic field to the outer midplane (OMP), at the top and at the OMP.

Fig. 13. Peak power at the divertor plate (a), helium ion density at the separatrix (b) and helium neutral flux across the separatrix to the core (c) vs. $\mathrm{p}_{\mathrm{DT}}$ for different locations of the 
gas puff. "trans" indicates incipient detachment of inner divertor (see text). 


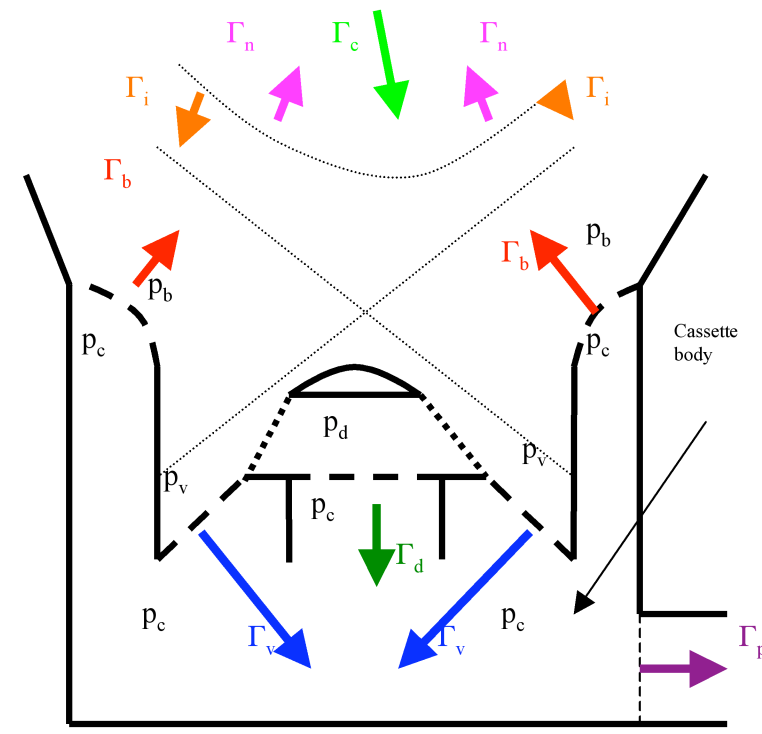

Fig. 1. Schematic of the particle flow in the divertor region of ITER 


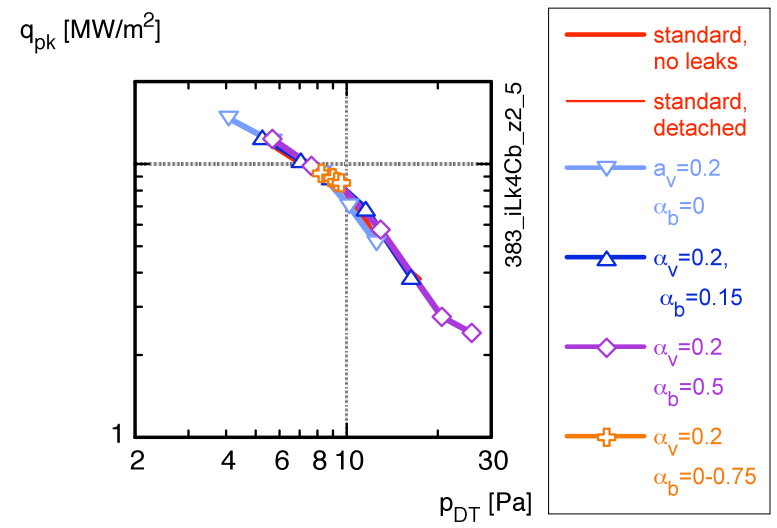

Fig. 2. Peak power load vs. $p_{\mathrm{DT}}$ for different values of the particle reflux fraction $\alpha_{\mathrm{b}}$. Two exploratory series of runs where one of $\alpha_{b}, \alpha_{v}$ was varied whereas the other and the total D content in the model were kept constant are also shown. 


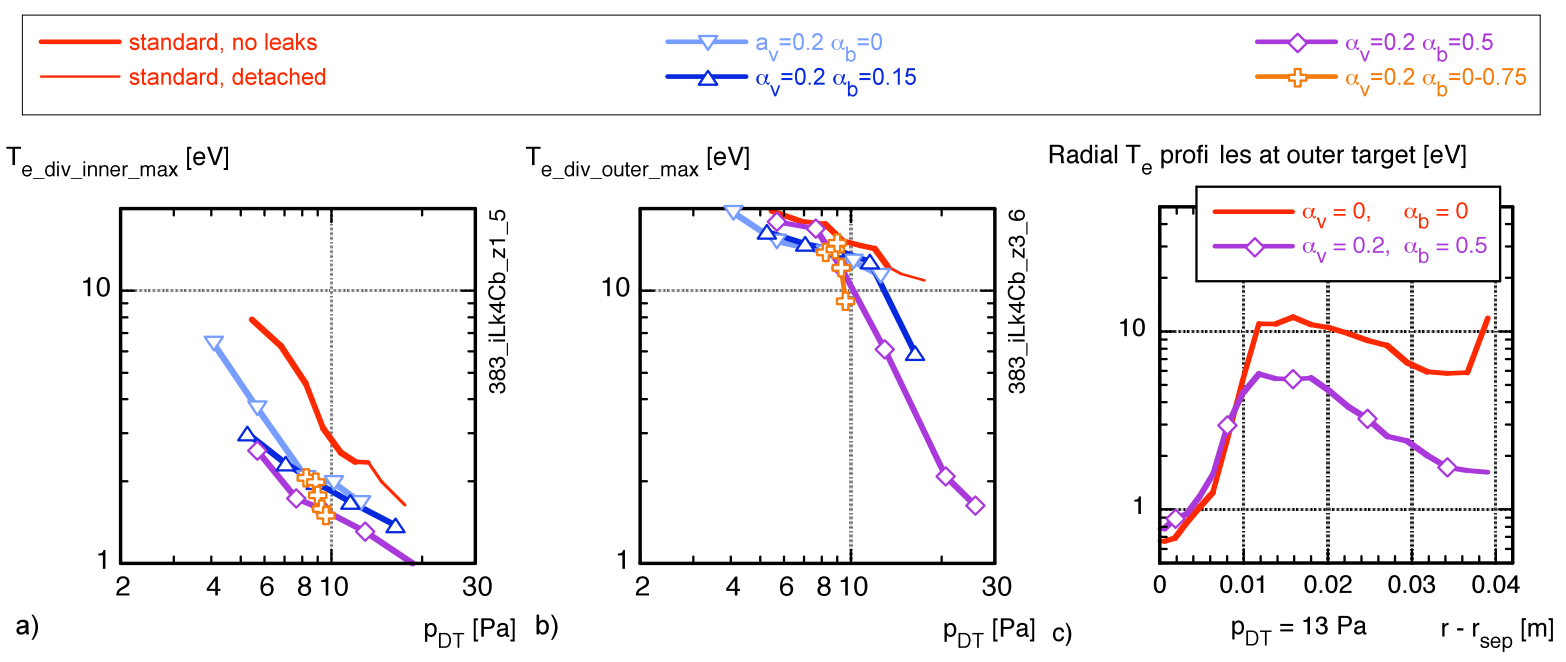

Fig. 3. Maximum electron temperature in the inner (a) and outer (b) divertor vs. $p_{D T}$ for different values of the particle reflux fraction $\alpha_{b}$. Fig. $3 c$ shows the radial profile of electron temperature at the outer target for $\mathrm{p}_{\mathrm{DT}}=13 \mathrm{~Pa}$. 


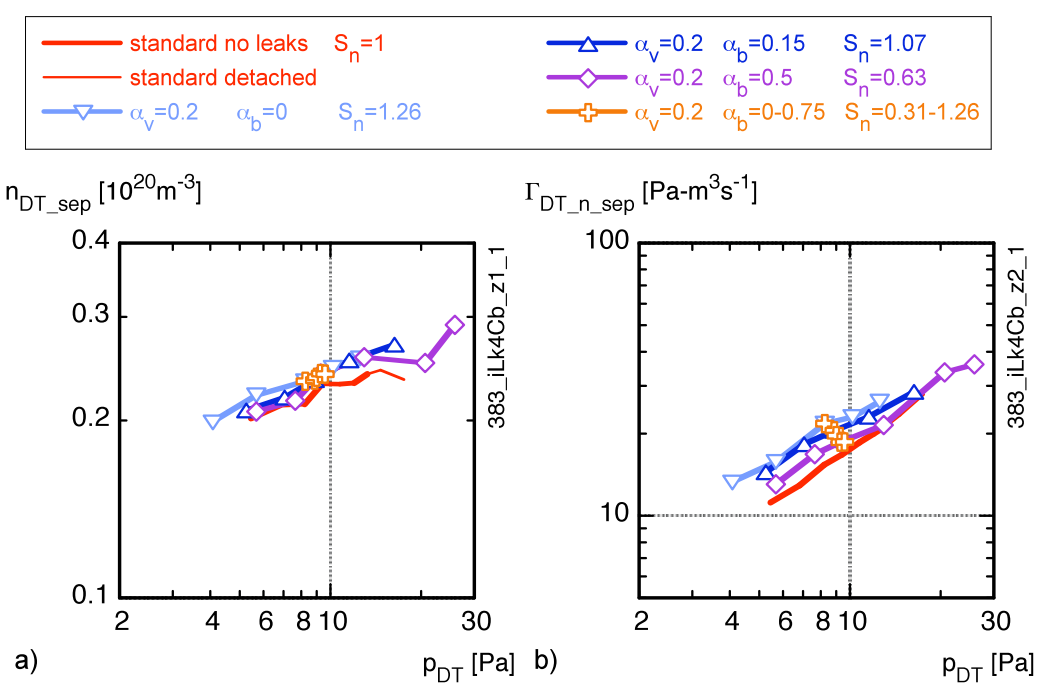

a)

$\Gamma_{\text {He_n_sep }}{ }^{\mathrm{f}} \mathrm{He}{ }^{-1} \mathrm{~S}_{\mathrm{n}}\left[\mathrm{Pa}-\mathrm{m}^{3} \mathrm{~s}^{-1}\right]$

$\mathrm{n}_{\mathrm{He} \_ \text {sep }} \mathrm{f}_{\mathrm{He}}{ }^{-1} \mathrm{~S}_{\mathrm{n}}\left[10^{20} \mathrm{~m}^{-3}\right]$

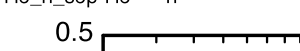
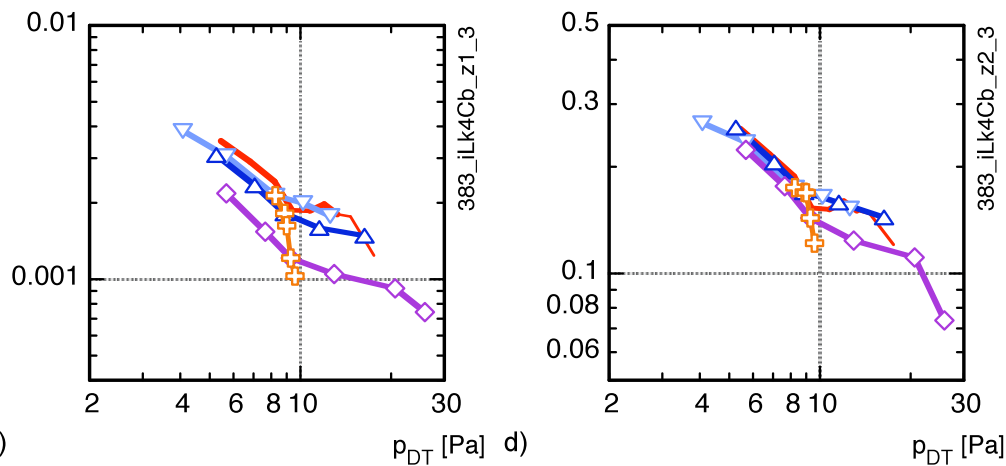

c)

$\left.\mathrm{p}_{\mathrm{DT}}[\mathrm{Pa}] \mathrm{d}\right)$

$\mathrm{p}_{\mathrm{DT}}[\mathrm{Pa}]$

Fig. 4. Ion density of D (a) and He (c), and neutral flux to the core of D (b) and He (d) vs. $p_{D T}$ for different values of particle reflux fraction $\alpha_{b}$ for the same series as Fig. 2 and 3. The helium-related quantities are scaled appropriately with the normalised effective pumping speed $S_{n}$ (see text and [8], values indicated in the legend). 


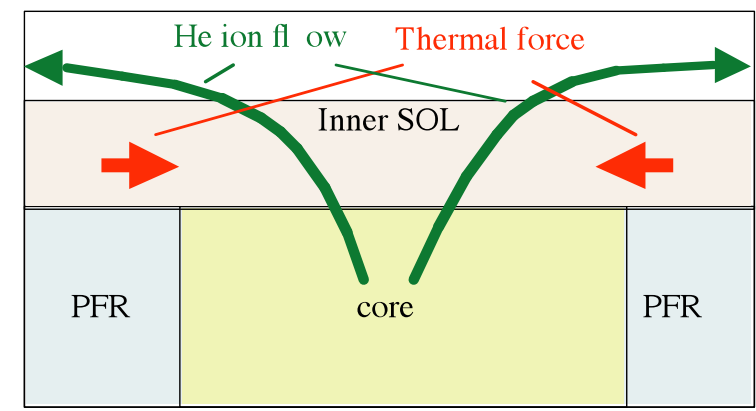

Fig. 5. Schematic of helium flow in the SOL and divertor region (unfolded on a rectangular area). Left and right edges represent the targets, upper edge the outer wall. 

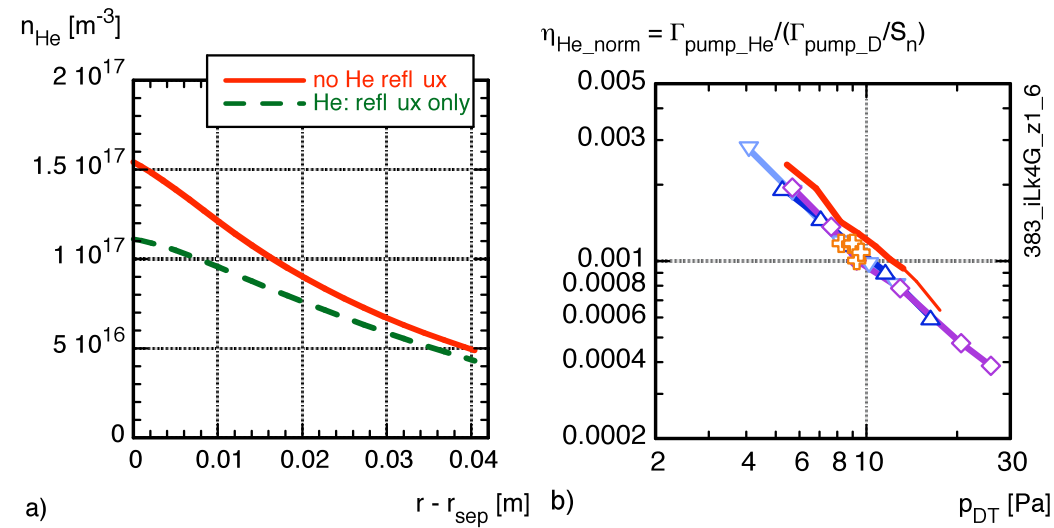

Fig. 6. (a) radial profiles of helium ion density in the SOL (outer mid-plane). (b) helium enrichment vs. $\mathrm{p}_{\mathrm{DT}}$ for different values of particle reflux fraction $\alpha_{\mathrm{b}}$. The enrichment is scaled appropriately with the normalised effective pumping speed $S_{n}$ (see text and [8]). Legend as in Fig. 4. 

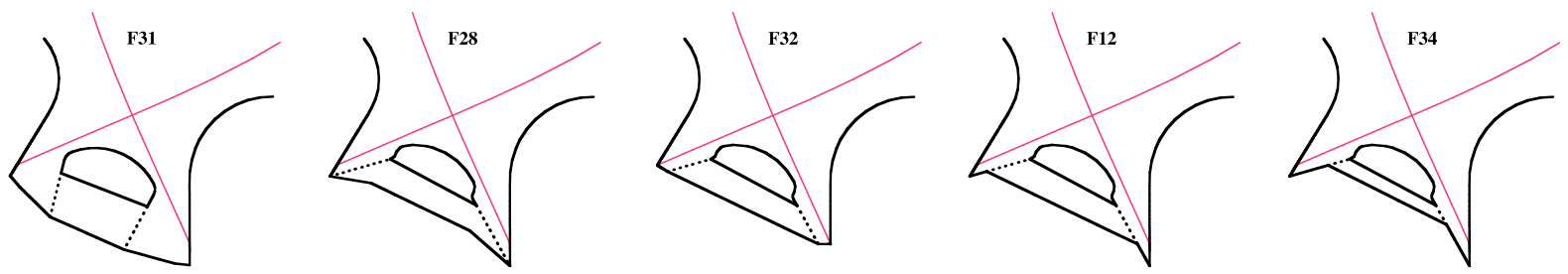

Fig. 7. Variation of divertor geometry used in the re-exploration on the effect of the "Vshapes" near the separatrix strike points. F12 marks our standard modelling configuration, F28, F31, and F32 are more open, and F34 more tight. Dotted lines indicate partially transparent surfaces at the entrance to the PFR. 


$\neg$ F31 flat bottom $\quad \longrightarrow$ F28 wide $V \quad \longrightarrow$ F32 strike at $V \quad$ F12 reference $\quad \longrightarrow$ F34 tightest $\mathrm{V}$

$\mathrm{q}_{\mathrm{pk}}\left[\mathrm{MW} / \mathrm{m}^{2}\right]$

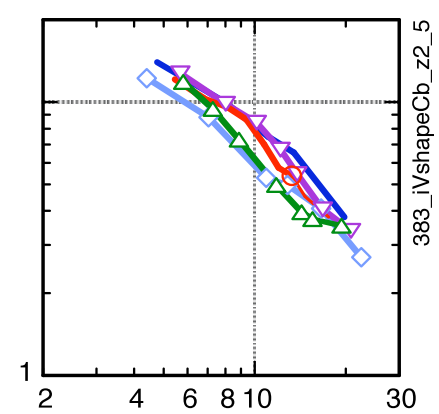

a) $\left.\mathrm{p}_{\mathrm{DT}}[\mathrm{Pa}] \mathrm{b}\right)$
$\Gamma_{\text {DT_n_sep }}\left[\mathrm{Pa}-\mathrm{m}^{3} \mathrm{~s}^{-1}\right]$

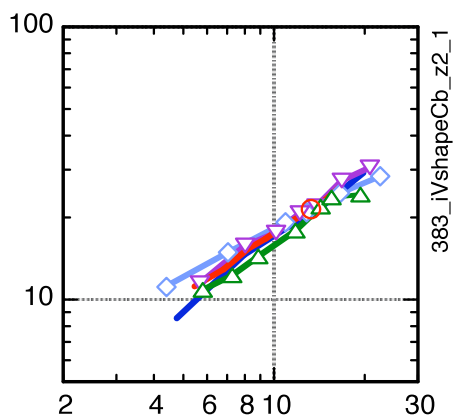

$\left.\mathrm{p}_{\mathrm{DT}}[\mathrm{Pa}] \mathrm{c}\right)$
$\mathrm{n}_{\mathrm{He} \_ \text {sep }} \mathrm{f}_{\mathrm{He}}{ }^{-1} \mathrm{~S}_{\mathrm{n}}\left[10^{20} \mathrm{~m}^{-3}\right]$

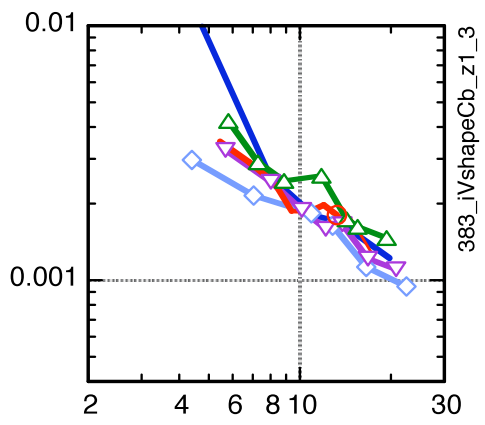

$\mathrm{p}_{\mathrm{DT}}[\mathrm{Pa}]$

Fig. 8. Peak power (a), D neutral influx into the core (b) and He ion density at the separatrix (c) vs. $\mathrm{p}_{\mathrm{DT}}$ for different shapes of the divertor bottom (see Fig. 7). $\mathrm{S}_{\mathrm{n}}=0.83$ for F32 and 1 for the other configurations. 


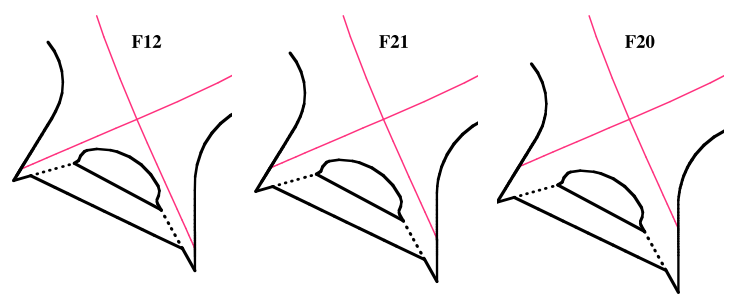

Fig. 9. Variation of the separatrix strike-point position on the targets used in the exploration. F12 is the reference geometry, F21 corresponds to an upward shift of $12 \mathrm{~cm}$ and F20 of 24 $\mathrm{cm}$. 


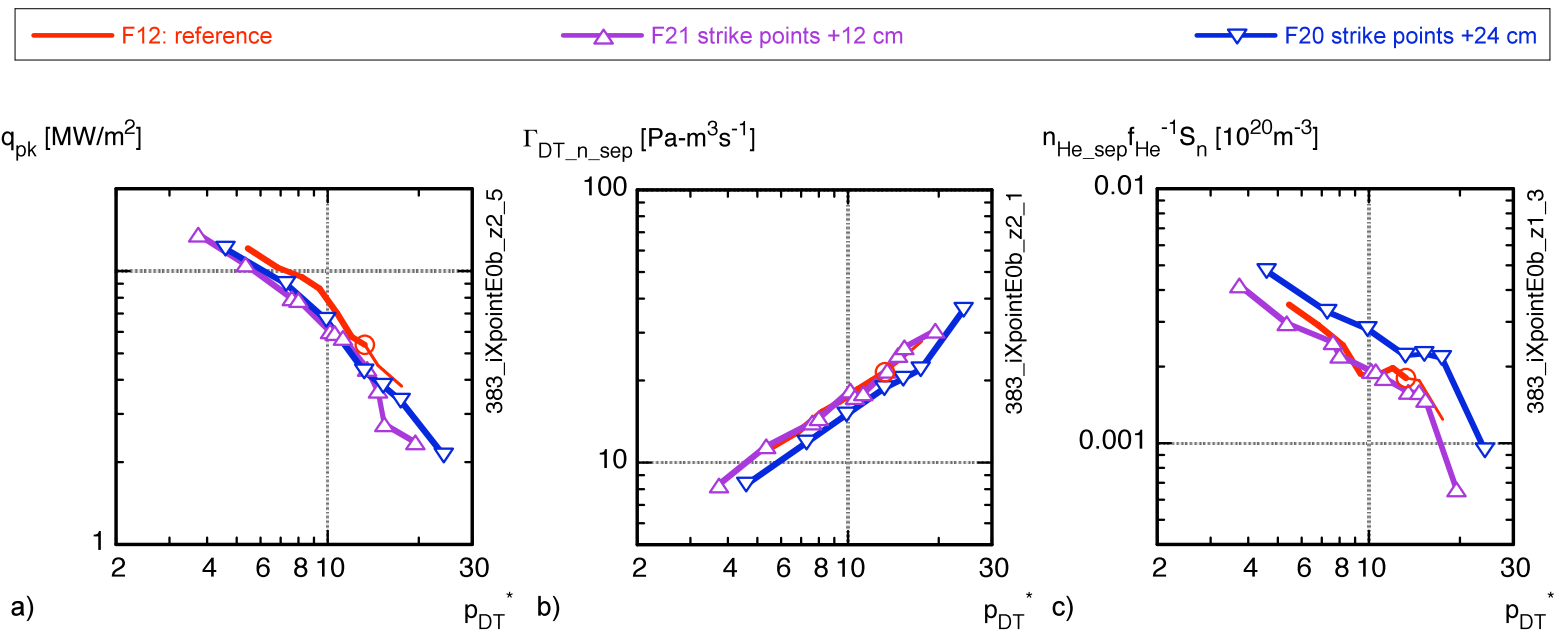

Fig. 10. Peak power (a), D neutral influx into the core (b) and He ion density at the separatrix (c) vs. normalised pressure in PFR, $\mathrm{p}_{\mathrm{DT}}{ }^{*}$, for different positions of the separatrix strike-points on the targets (see Fig. 9). 


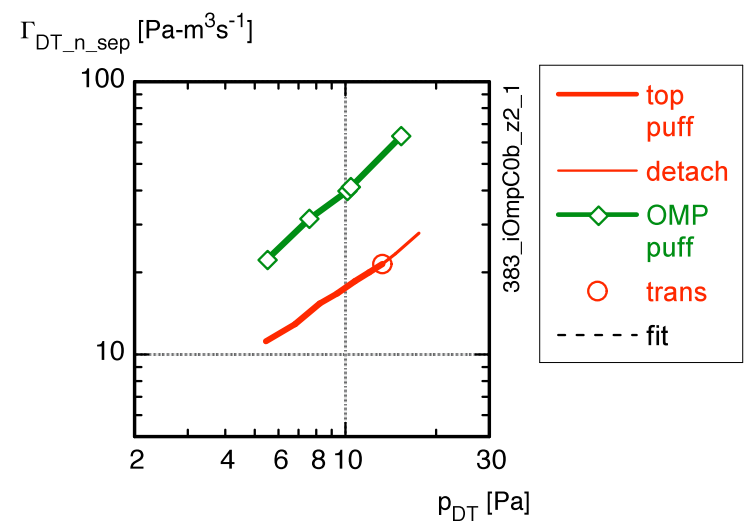

Fig. 11. Influx of $D$ neutrals into the core (core fuelling rate) vs. $\mathrm{p}_{\mathrm{DT}}$ for different locations of the gas puff. "trans" indicates incipient detachment of inner divertor (see text). 

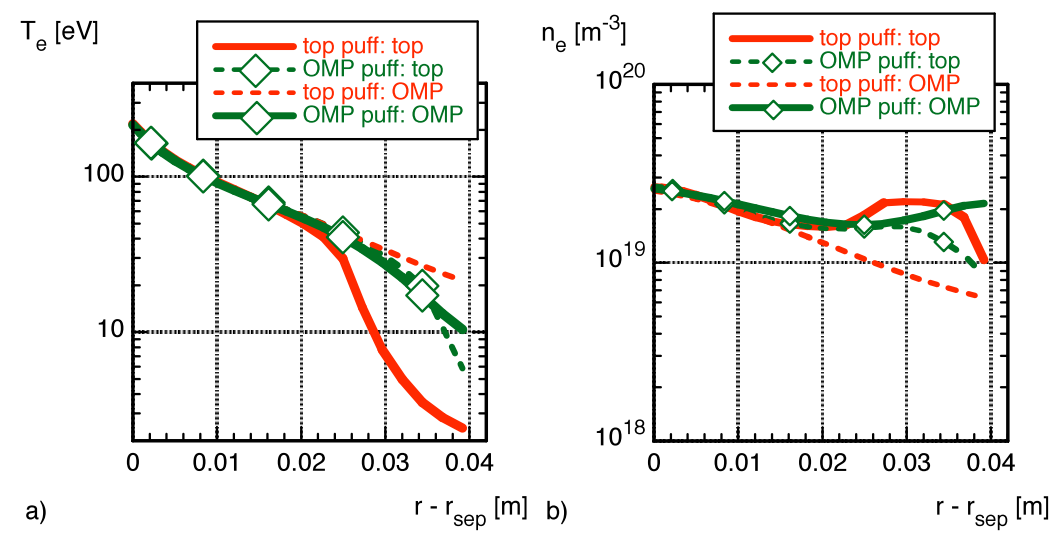

Fig. 12 Radial profiles of the electron temperature (a) and electron density (b), mapped along the magnetic field to the outer midplane (OMP), at the top and at the OMP. 


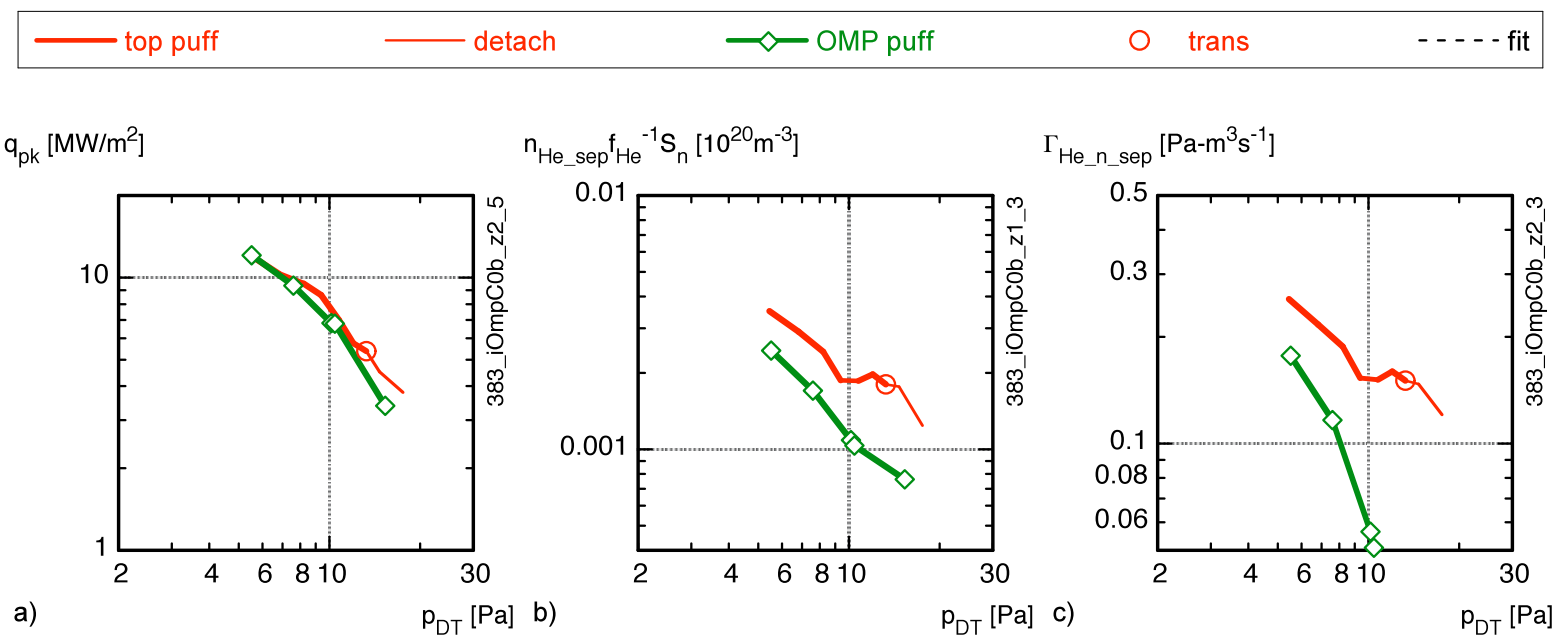

Fig. 13. Peak power at the divertor plate (a), helium ion density at the separatrix (b) and helium neutral flux across the separatrix to the core (c) vs. $\mathrm{p}_{\mathrm{DT}}$ for different locations of the gas puff. "trans" indicates incipient detachment of inner divertor (see text). 